\title{
Electromagnetically induced interference in a superconducting flux qubit
}

\author{
Lingjie $\mathrm{Du}^{1,2}$, Yang $\mathrm{Yu}^{1}$, and Dong $\operatorname{Lan}^{1}$ \\ ${ }^{1}$ National Laboratory of Solid State Microstructures and Department of Physics \\ Nanjing University, Nanjing 210093, China \\ E-mail: lingjie.du@rice.edu \\ yuyang@nju.edu.cn \\ ${ }^{2}$ Department of Physics and Astronomy, Rice University, Houston, Texas 77251-1892, USA
}

Received November 23, 2012, revised December 22, 2012

\begin{abstract}
Interaction between quantum two-level systems (qubits) and electromagnetic fields can provide additional coupling channels to qubit states. In particular, the interwell relaxation or Rabi oscillations, resulting, respectively, from the multi- or single-mode interaction, can produce effective crossovers, leading to electromagnetically induced interference in microwave driven qubits. The environment is modeled by a multimode thermal bath, generating the interwell relaxation. Relaxation induced interference, independent of the tunnel coupling, provides deeper understanding to the interaction between the qubits and their environment. It also supplies a useful tool to characterize the relaxation strength as well as the characteristic frequency of the bath. In addition, we demonstrate the relaxation can generate population inversion in a strongly driving two-level system. On the other hand, different from Rabi oscillations, Rabi-oscillation-induced interference involves more complicated and modulated photon exchange thus offers an alternative means to manipulate the qubit, with more controllable parameters including the strength and position of the tunnel coupling. It also provides a testing ground for exploring nonlinear quantum phenomena and quantum state manipulation in qubits either with or without crossover structure.
\end{abstract}

PACS: 03.67.Lx Quantum computation architectures and implementations;

32.80.Xx Level crossing and optical pumping;

42.50.Hz Strong-field excitation of optical transitions in quantum systems; multiphoton processes; dynamic Stark shift.

Keywords: quantum qubits, Rabi-oscillation-induced interference.

\section{Introduction}

Strongly driven transition at avoided crossings is an old issue but plays an essential role in a variety of physical phenomena. Cases of avoided crossings can be met in many areas in physics, such as atomic-collision [1,2], laser-atom interactions [3], optical atoms [4], ultracoldmolecules gas [5], Bose-Einstein condensate [6], and more recently, qubits for quantum information processing [7-9]. A qubit is generally built from a quantum two-level system (TLS), which is adjustable by external control fields. If the external control parameter is changed so that a TLS traverses the crossover, a non-adiabatic transition between two states occurs. This transition is known as LandauZener (LZ) transition [10], which can be used to enhance the quantum tunneling rate $[11,12]$, to prepare the quantum states [13], and to control the gate operations [14]. When the qubit is subjected to a strong periodic microwave field, consecutive LZ transitions between two states at the crossover may result in Landau-Zener-Stückelberg (LZS) interference $[15,16]$, which has been demonstrated in many systems [17-28]. The strong driving could allow for fast and reliable control of the qubits, paving a way of demonstration of macroscopic quantum coherence and implementation of practical quantum processor.

In practice, besides controlling fields, TLSs inevitably couple with electromagnetic environments which can often be described as a multimode thermal bath of harmonic oscillators [29], experiencing dephasing and energy relaxation simultaneously. Both processes contribute to decoherence which may hinder qubit performance in quantum information [30-34]. In general, the bath couples to the qubit longitudinally, causing the dephasing related with the environment property at low energies. The transverse inte- 
raction between the qubit and bath produces the interwell relaxation between two lowest qubit states. In previous works [16,35-37], the interwell relaxation is described empirically and we will show additional interference is usually overlooked. Therefore, the investigation on strongly driven dynamics considering additional electromagnetic degrees of freedom will clarify the environmental effect on qubits. Furthermore, it is possible to improve the quantum gate operation with LZS interference.

In this article, we study the strongly microwave driven qubit in the presence of additional electromagnetic interactions based on the general theories of TLSs [36-45]. It is found that the interactions between qubits and electromagnetic fields provide additional coupling channels to qubit states, which will detour the evolution path to lead a new mechanism of interference. We name the new mechanism of interference as electromagnetically induced interference (EII). Two typical electromagnetic systems: (i) a thermal bath with multimode electromagnetic fields and (ii) a weak single-mode field, are chosen to discuss interesting phenomena and applications.

We show that the relaxation induced coupling contributes to asymmetrical transitions to form interferences, i.e. relaxation induced interference (RII). We find that the relaxation can generate population inversion in a strongly driven TLS through the interference, which violates the intuitive picture. This work also clarifies the range of validity of previous results in atomic physics. Moreover, the model presents deeper understanding to the effect of the environment. It provides a useful tool for characterizing the interaction of qubits and environment, e.g., the relaxation strength and the characteristic frequency of the bath.

Since the interwell relaxation is determined by the environment, we turn to a more controllable case by applying a weak single mode field which results Rabi oscillations. Rabi oscillation can construct new controllable coupling channels which act as effective level crossover. The interference, which we call Rabi-oscillation-induced interference (ROII), is generated under the strong microwave field. ROII offers a fully tunable way to operate qubits in the strongly driven region. It is promising for further application in the time-dependent interference which can manipulate the qubit over short timescales. In addition, it also supplies a testing ground for exploring nonlinear quantum phenomena and quantum state manipulation, in the general TLS without the crossover structure.

The paper is organized as follows. In Sec. 2, through the perturbation theory, we calculate transition rates based on the relaxation induced coupling. Then we present a discussion about relaxation induced interference. In Sec. 3, we discuss the competition of two interferences, one is RII and another is the primary LZS interference. By tuning the relaxation strength and the characteristic frequency of environment bath, we show different interference patterns in saturated and unsaturated cases. Finally, we compare our model with the phenomenological relaxation theory. In Sec. 4, we study Rabi-oscillation-induced interference in the presence of the weak monochrome field. Then we discuss new interference mechanism and its application in quantum state manipulation. In Sec. 5, we discuss EII in superconducting qubits.

\section{Relaxation induced interference}

We consider a two-level system described by the Hamiltonian in the presence of the environment bath with a continuum of electromagnetic modes in equilibrium at temperature $T$ (we set $\hbar=k_{B}=1$ )

$$
\begin{gathered}
H=-\frac{\Delta}{2} \sigma_{x}-\frac{\varepsilon(t)}{2} \sigma_{z}-\frac{1}{2} \sum \lambda_{i}\left(a_{i}^{\dagger}+a_{i}\right) \sigma_{z}- \\
-\frac{\phi}{2} \sum \lambda_{i}\left(a_{i}^{\dagger}+a_{i}\right) \sigma_{x}+H_{B},
\end{gathered}
$$

where $\sigma_{X}$ and $\sigma_{z}$ are Pauli matrices, $\varepsilon(t)=\varepsilon_{0}+A \cos \omega t$, $\sigma_{z}=|1\rangle\langle 1|-| 0\rangle\left\langle 0\left|, \sigma_{X}=\right| 1\right\rangle\langle 0|+| 0\rangle\langle 1| . H_{B}=\sum \omega_{i}\left(a_{i}^{\dagger} a_{i}+1 / 2\right)$ is the bath Hamiltonian, $a_{i}^{\dagger}$ and $a_{i}$ denote the boson creation and annihilation operators corresponding to the frequency $\omega_{i}$ for the bath, and $\lambda_{i}$ expresses the oscillator coupling constant. $\phi$ represents the transverse interaction strength. It should be mentioned that Eq. (1) can be applied in a general TLS. If there is no crossover structure, the tunnel coupling $\Delta$ becomes zero.

Here, we replace the conventional expressions with the noise operator model [36-39,46], where the environment noise is assumed to follow the Gaussian approximation. $Q=\sum \lambda_{i}\left(a_{i}^{\dagger}+a_{i}\right)$ is the environment operator, $-\phi Q \sigma_{x} / 2$ describes the transverse interaction between the qubit and the multimode fields (the interwell relaxation) that causes the energy exchange between the qubit and bath. $-Q \sigma_{z} / 2$ describes the longitudinal interaction between the qubit and the multimode fields (the dephasing process) dominated by the low-frequency energy from the bath. As a result all averages can be expressed by the spectral density

$$
S\left(\omega^{\prime}\right)=\frac{1}{2 \pi} \int_{-\infty}^{\infty} d t e^{i \omega^{\prime} t}\langle Q(t) Q(0)\rangle .
$$

Now we follow the process in Ref. 36. By taking an interaction picture with respect to $H_{B}$, the Hamiltonian is changed to

$$
H_{1}=-\frac{\Delta}{2} \sigma_{X}-\frac{\varepsilon(t)}{2} \sigma_{z}-\frac{Q(t)}{2} \sigma_{z}-\frac{\phi Q(t)}{2} \sigma_{X},
$$

where $Q(t)=\mathrm{e}^{i H_{B} t} Q \mathrm{e}^{-i H_{B} t}$. Then we make a transformation to a rotation frame, such that a state vector $|\psi\rangle$ in the interaction picture can be expressed as $|\psi\rangle=U_{0}(t)\left|\psi^{\prime}\right\rangle$, with

$$
U_{0}(t)=\mathcal{T} \exp \left\{\frac{i}{2} \sigma_{z} \int_{0}^{t}[Q(\tau)+\varepsilon(\tau)] d \tau\right\}
$$


(a)

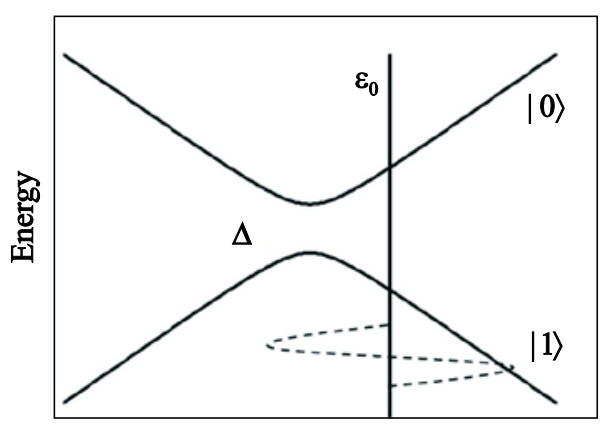

Energy detuning

(b)

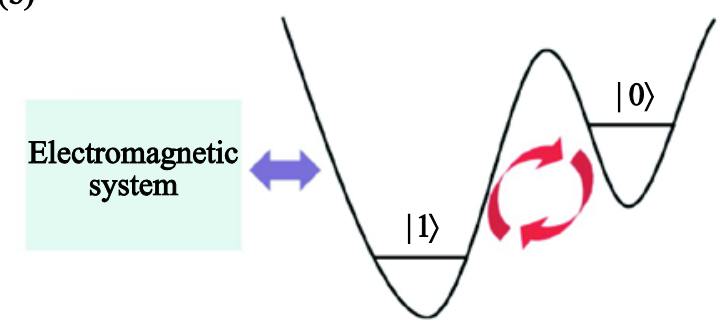

Fig. 1. (Color online) (a) Schematic energy diagram of a driven two-level system. The dotted curve represents the strong driving field $A \cos \omega t$. The field through the tunnel coupling $\Delta$ forms a LZS interference, exchanging photons with the qubit. (b) Quantum tunnel coupling exists between states $|0\rangle$ and $|1\rangle$. The interaction between a qubit and an electromagnetic system (such as the environment bath or a single-mode electromagnetic field) would form new couplings between the two states.

where $\mathcal{T}$ expresses the time ordering and $\left|\psi^{\prime}\right\rangle$ is the state vector in the rotation frame.

Here we get the effective Hamiltonian

$$
\begin{aligned}
H^{\prime}(t) & =-\frac{1}{2} \exp \left\{-i \int_{0}^{t} \varepsilon(\tau) d \tau\right\}\left[\Delta U_{+}^{\dagger}(t) U_{-}(t)+\right. \\
& \left.+\phi U_{+}^{\dagger}(t) Q(t) U_{-}(t)\right]|1\rangle\langle 0|+\text { H.c. },
\end{aligned}
$$

where

$$
U_{ \pm}(t)=\mathcal{T} \exp \left[ \pm \frac{i}{2} \int_{0}^{t} Q(\tau) d \tau\right] .
$$

Then we obtain the total density matrix of the qubit system plus the bath at the time interval $t, \rho_{S B}(t)=U_{1}(t, 0) \times$ $\times \rho_{S B}(0) U_{1}^{\dagger}(t, 0)$, where the time evolution operator in the laboratory frame can be expressed as

$$
\begin{array}{r}
U_{1}\left(t_{1}, t_{2}\right)=\mathrm{e}^{-i H_{B} t_{1}} U_{0}\left(t_{1}\right) U_{V}\left(t_{1}, t_{2}\right) U_{0}^{\dagger}\left(t_{2}\right) \mathrm{e}^{i H_{B} t_{2}}, \\
\text { and } \quad U_{V}\left(t_{1}, t_{2}\right)=\mathcal{T} \exp \left[-i \int_{t_{2}}^{t_{1}} d t^{\prime} H^{\prime}\left(t^{\prime}\right)\right] .
\end{array}
$$

The system reduced density matrix is $\rho(t)=\operatorname{Tr}_{B}\left[\rho_{S B}(t)\right]$, where $\operatorname{Tr}_{B}[\ldots]$ denotes the trace over the environmental degrees of freedom. When the system-bath interaction energy is small, we assume $\rho_{S B}(0)$ factors as a direct product $\rho_{S B}(0)=\rho(0) \otimes \rho_{B}$, where $\rho_{B}=\mathrm{e}^{-H_{B} / T}$ is the density matrix of the bath.

Due to the weak relaxation and tunnel coupling compared with the dephasing rate $\Gamma_{2}$, we consider the time interval $t$ within which the change of qubit population is slow, but $t$ is much larger than the dephasing time $T_{2}=1 / \Gamma_{2}$. Then we make the approximation with $U_{V}(t, 0)$ by performing a perturbation expansion:

$$
U_{V}(t, 0) \approx 1-i \int_{0}^{t} d t^{\prime} H^{\prime}\left(t^{\prime}\right) .
$$

Strong dephasing eliminates the off-diagonal elements of $\rho(t)$ quickly and changes the density matrix equation to the rate equation. Considering the small time interval, i.e. $\dot{\rho}_{00}(t) t \sim 0$, if the noise is frequency independent (white) in low-frequency region, we have the rate equation (see the Appendix)

$$
\dot{\rho}_{00}(t)=W_{10}+\Gamma_{10},
$$

where quantum tunneling $\Delta$ resulted Landau-Zener transition rate from state $|1\rangle$ to $|0\rangle$ is

$$
W_{10}=\frac{\Delta^{2}}{2} \sum_{n} \frac{\Gamma_{2} J_{n}^{2}(A / \omega)}{\left(\varepsilon_{0}+n \omega\right)^{2}+\Gamma_{2}^{2}},
$$

and $W_{01}\left(\varepsilon_{0}\right)=W_{10}\left(-\varepsilon_{0}\right)$, where $\Gamma_{2}=\pi S(0)$. On the other hand, the transition rate induced by the interwell relaxation from state $|1\rangle$ to $|0\rangle$ is

$$
\Gamma_{10}=\frac{\phi^{2}}{4} \sum_{n} J_{n}^{2}\left(\frac{A}{\omega}\right) S\left(-\varepsilon_{0}-n \omega\right),
$$

and $\Gamma_{01}\left(\varepsilon_{0}\right)=\Gamma_{10}\left(-\varepsilon_{0}\right)$. It is clear that the dephasing would not influence the transition in Eq. (7). In order to clarify the physical picture, we consider an important class of spectral densities $[29,45]$

$$
S\left(\omega^{\prime}\right)=\frac{\alpha \omega^{\prime} \mathrm{e}^{-\mid \omega^{\prime} / \omega_{C}}}{1-\mathrm{e}^{-\omega^{\prime} / T}} .
$$

The spectral density at negative (positive) frequencies corresponds to the emission (absorption) of photons to (from) the bath. Generally, the spectral density describes the ability of energy exchange between the qubit and bath.

First of all we consider a special situation in Eq. (7), where the microwave amplitude $A=0$, then Eq. (7) is changed to the downward and upward relaxation rates

$$
\Gamma_{01}=\frac{\phi^{2}}{4} S\left(\varepsilon_{0}\right), \Gamma_{10}=\frac{\phi^{2}}{4} S\left(-\varepsilon_{0}\right) .
$$

Seen from the form of $S\left(\omega^{\prime}\right)$, the bath satisfies the Einstein relation with a larger downward relaxation rate

$$
\Gamma_{10} / \Gamma_{01}=\mathrm{e}^{-\varepsilon_{0} / T}
$$

$\Gamma_{01}\left(\Gamma_{10}\right)$ corresponds to the process that qubit is spontaneously relaxed down (up) from state $|0\rangle$ to $|1\rangle$ (from 
state $|1\rangle$ to $|0\rangle$ ) accompanied by emitting (absorbing) photons with energy $\varepsilon_{0}$ to (from) environment bath. Equation (8) demonstrates that for the low temperature $T \lesssim 0.5 \omega_{c}$ there is a maximum downward relaxation rate near the detuning $\varepsilon_{0}= \pm \omega_{c}$. Then at the characteristic frequency $\omega_{c}$, the bath can absorb photons from the qubit fastest. For the high temperature, the downward relaxation rate will decrease monotonically with the detuning. From Eqs. (8) and (10), we can know with the detuning approaching to zero, relaxation rates become constant and equal, while with the detuning larger than $\omega_{c}$, transition rates gradually become zero. Therefore even if the temperature does not satisfy the relation $T \lesssim 0.5 \omega_{c}$, there still exist the downward relaxation and smaller upward relaxation, mostly near the appropriate detuning $\varepsilon_{0}= \pm \omega_{c}$. Hence the interwell relaxations can asymmetrically couple two states in the qubit at the detuning $\varepsilon_{0} \approx \pm \omega_{c}$. Serving as beam splitters, the relaxations at the detuning $\varepsilon_{0} \approx-\omega_{C}$ can split state $|1\rangle$ with a larger possibility than $|0\rangle$ as indicated in Fig. 2(a).

In the presence of microwave, i.e. $A \neq 0$, the stimulated transition will change the population distribution. Substituting $S\left(\omega^{\prime}\right)$ in Eq. (8) into Eq. (7), we get

$$
\Gamma_{01}=\frac{\phi^{2} \alpha}{4} \sum_{n} J_{n}^{2}\left(\frac{A}{\omega}\right) \frac{\left(\varepsilon_{0}-n \omega\right) \mathrm{e}^{-\left|\varepsilon_{0}-n \omega\right| / \omega_{C}}}{1-\mathrm{e}^{-\left(\varepsilon_{0}-n \omega\right) / T}} .
$$

The term $\Gamma_{01}\left(\Gamma_{10}\right)$ can be understood from Fig. 2, where the qubit state decaying (exciting) processes with $\Gamma_{01}\left(\Gamma_{10}\right)$ are shown. For the decaying process in Fig. 2(a), the qubit emits a photon with energy $\varepsilon_{0}$. Part of this energy is absorbed by the microwave field and the residue is absorbed by the bath. If the energy absorbed by the field is larger than $\varepsilon_{0}$, i.e. $n \omega>\varepsilon_{0}$, the bath would emit photons with energy $n \omega-\varepsilon_{0}$ to the field (not notable in Fig. 2). $\Gamma_{01}$ reaches maximum when the energy $\varepsilon_{0}$ is larger than the energy absorbed the field, and the residue energy absorbed by the bath is just $\omega_{c}$. It is similar to the exciting process in Fig. 2(b). $\Gamma_{10}$ reaches maximum when the energy provided by the field is larger than the energy $\varepsilon_{0}$, and the residue energy absorbed by bath is just $\omega_{c}$.

Furthermore, Eqs. (7) and (11) show that the microwave amplitude can modulate the transition through the asymmetric couplings to form interference. In order to reveal the physical picture, we further simplify the spectral density in Eq. (8) as

$$
S\left(\omega^{\prime}\right)=\left\{\begin{array}{cc}
\alpha \omega_{c}, & \omega^{\prime}=\omega_{c} \\
0, & \omega^{\prime} \neq \omega_{c} .
\end{array}\right.
$$

It is clear that here the bath can only absorb photons with the characteristic frequency $\omega_{c}$. Moreover there is only downward relaxation, which means the splitters at $\varepsilon_{0}=\omega_{c}$ or $-\omega_{c}$ will work only for $|0\rangle$ or $|1\rangle$, respectively. Substituting Eq. (12) into Eq. (7), we obtain transition rates
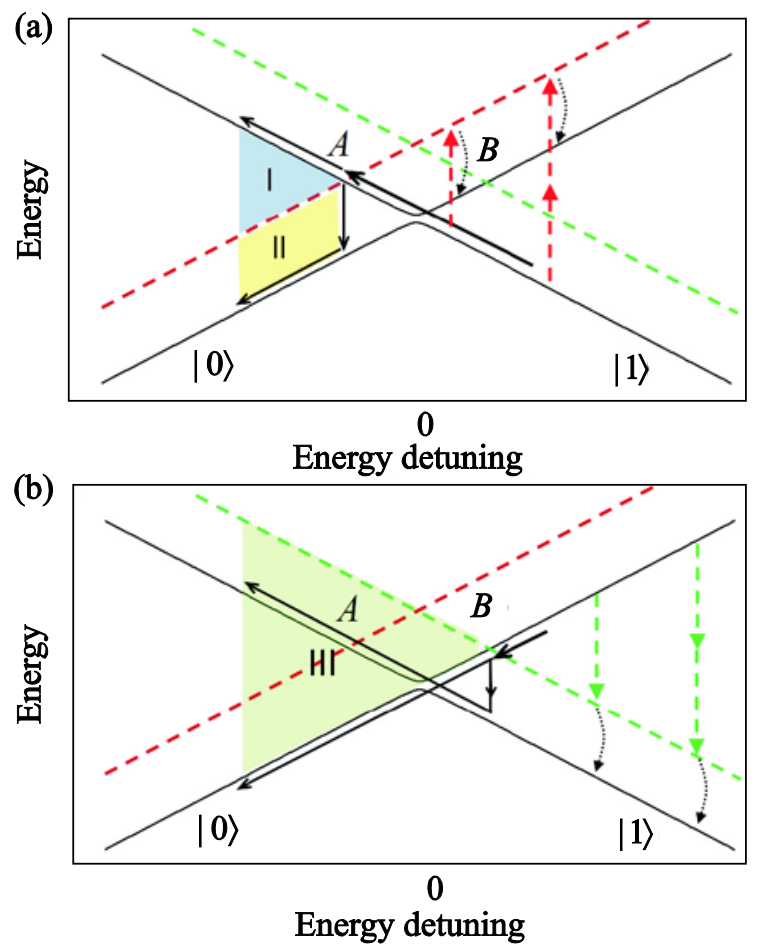

Fig. 2. (Color online) Schematic energy diagram of relaxation induced interference: (a) refers to the transition from state $|1\rangle$ to $|0\rangle$ through $A$; (b) refers to the transition from state $|0\rangle$ to $|1\rangle$ through $B$. The dashed red upward arrows mark the multiphoton absorption from the driving microwave field while the dashed green downward arrows mark the multiphoton release to the microwave. The dotted arrows describe the energy released or absorbed by the bath. Effectively, the resonant conditions are $n \omega=\varepsilon+\omega_{C}$ (from state $|1\rangle$ to $|0\rangle$ ) and $n \omega=\varepsilon-\omega_{C}$ (from state $|0\rangle$ to $|1\rangle)$. The blue region I describes effective phase difference contributing to the interference at $A$. The yellow region II expresses the phase eliminated by the bath. The green region III expresses the effective phase difference contributing to the interference at $B$.

$$
\begin{gathered}
\Gamma_{01}=\frac{\phi^{2} \alpha \omega_{c}}{4}\left\{\begin{array}{cc}
J_{n}^{2}\left(\frac{A}{\omega}\right), & \varepsilon_{0}=\omega_{c}+n \omega, \\
0, & \varepsilon_{0} \neq \omega_{c}+n \omega,
\end{array}\right. \\
\Gamma_{10}=\frac{\phi^{2} \alpha \omega_{C}}{4}\left\{\begin{array}{cc}
J_{n}^{2}\left(\frac{A}{\omega}\right), & \varepsilon_{0}=-\omega_{C}+n \omega, \\
0, & \varepsilon_{0} \neq-\omega_{c}+n \omega .
\end{array}\right.
\end{gathered}
$$

At the detuning $\varepsilon_{0}=n \omega \pm \omega_{C}$, transition rates with the probability $\phi^{2} \alpha \omega_{c} J_{n}^{2}(A / \omega) / 4$ are modulated by the amplitude. As shown in Fig. 2(a), when the system is initialized in state $|1\rangle$, the microwave sweeps the state along $|1\rangle$ until it reaches the detuning $A$ with $\varepsilon_{0}=-\omega_{c}$ at time $t_{1}$. Then state $|1\rangle$ is split into the superposition of states $|0\rangle$ and $|1\rangle$, collecting different phases in two paths. After evolving independently, they are driven back to $A$ (time $t_{2}$ ). $|1\rangle$ is split into $|0\rangle$ and $|1\rangle$ again, while $|0\rangle$ is not split. The relative phase difference accumulated by states $|0\rangle$ and $|1\rangle$ 
(marked with the blue region I plus yellow region II) results in the interference. We call this case relaxation induced interference.

However, according to the simplified spectral densities in Eq. (12), the second term in Eq. (A.10) in Appendix can be changed to

$$
\frac{\phi^{2} \alpha}{4} \int_{0}^{t} d t_{1} \int_{0}^{t} d t_{2} \int d \omega^{\prime} \sum_{n} J_{n}^{2}\left(\frac{A}{\omega}\right) \mathrm{e}^{i\left(\varepsilon_{0}+n \omega+\omega_{C}\right)\left(t_{1}-t_{2}\right)},
$$

which describes that the multimode fields transversely produce a phase accumulation $i \omega_{c} t$ in the transition from state $|1\rangle$ to $|0\rangle$. Then the effective phase difference contributing to the interference at $A$ is $\Delta \theta_{q a}$ (blue region I) since the bath eliminates part of the phase (yellow region II). At $A$, the interference phase

$$
\Delta \theta_{q a}=\int_{t_{1}}^{t_{2}} d t\left(\varepsilon_{|0\rangle}-\varepsilon_{|1\rangle}+\omega_{c}\right)
$$

where $\varepsilon_{|1\rangle}=-\varepsilon(t) / 2$ and $\varepsilon_{|0\rangle}=\varepsilon(t) / 2$, is modulated by the amplitude. The interference condition $\Delta \theta_{q a}=2 \pi n$ or $2 \pi n+\pi$ would make the interference effect maximum or minimum. The total phase gained over one period referring to $A$ is

$$
\theta_{A}=\oint d t\left(\varepsilon_{|0\rangle}-\varepsilon_{|1\rangle}+\omega_{C}\right)=2 \pi\left(\varepsilon_{0}+\omega_{C}\right) / \omega .
$$

Then the qubit state is driven to the detuning $B$ where $\varepsilon_{0}=\omega_{c}$ and a similar procedure happens with an effective phase difference contributing to the interference at $B$ (green region III). The total phase gained over one period referring to $B$ is

$$
\theta_{B}=\oint d t\left(\varepsilon_{|1\rangle}+\omega_{C}-\varepsilon_{|0\rangle}\right)=2 \pi\left(-\varepsilon_{0}+\omega_{C}\right) / \omega .
$$

The stable interference can be constructed with the resonant condition $\theta_{A}$ or $\theta_{B}=2 \pi n$. It should be mentioned that this resonant condition is different from the interference condition, playing the role of stabilizing the interference effect. Therefore, the resonant transition from state $|1\rangle$ to $|0\rangle$ (from state $|0\rangle$ to $|1\rangle$ ) would occur with the relation shown in Eq. (13). For the actual spectral density as shown in Eq. (8), there would be additional interference resonant peak width which is on the order of $\omega_{c}$. Therefore, in order to form clear interference fringes, the microwave frequency should be larger than the characteristic frequency.

Generally, the interwell relaxation provides additional couplings between states $|0\rangle$ and $|1\rangle$ to form extra interferences. In the presence of tunnel coupling $\Delta$, together with the primary LZS interference, the two ones would simultaneously determine the final qubit population. In addition, due to asymmetrical upward and downward relaxation transitions, there are interesting phenomena which will be discussed in next section.

\section{Competition of two interferences}

In order to study RII clearly, firstly we assume there is no crossover structure, i.e. $\Delta=0$, to separate this interference from the primary one. Then the stationary population in state $|0\rangle$ in RII is

$$
S=\frac{\Gamma_{10}\left(\varepsilon_{0}\right)}{\Gamma_{10}\left(\varepsilon_{0}\right)+\Gamma_{01}\left(\varepsilon_{0}\right)},
$$

where $\Gamma_{01}$ and $\Gamma_{10}$ are determined by Eq. (11). In the case $\omega \gg \omega_{c}$, at the detuning $\varepsilon_{0}=n \omega-\omega_{C}$, multiphoton resonant fringes of the transition rate $\Gamma_{10}\left(\varepsilon_{0}\right)$ are observable in Fig. 3(a). Due to asymmetric transition rates, population inversion also emerges. The modulation of the amplitude results that interference fringes are the Bessel dependence on the driving amplitude. Moreover, the peak width of spectral density yields different fringes from those of the primary LZS interference. In the other case $\omega<\omega_{c}$, the resonant peak width in RII is larger than $\omega$. Therefore, the individual resonances and Bessel function dependence are no longer distinguishable and merge into a continuous band [Fig. 3(b)]. Population inversion also vanishes. In Fig. 3(a) the temperature is larger than the characteristic frequency while in Fig. 3(c) the temperature is much smaller than the characteristic frequency. Besides, although in the high temperature there is not maximum downward relaxation, it is obvious that patterns in Fig. 3(a) and (c) are similar, which agrees the discussion in Sec. 3 .

\subsection{Population dynamics}

Then we return to the case in the presence of $\Delta$. In order to describe the time evolution of the qubit population, we employ rate equations, in which the population obey

$$
\begin{gathered}
-\left(W_{01}+\Gamma_{01}\right) \rho_{00}(t)+\left(W_{10}+\Gamma_{10}\right) \rho_{11}(t)=\dot{\rho}_{00}(t), \\
\rho_{00}(t)+\rho_{11}(t)=1,
\end{gathered}
$$

where $W_{01}$ and $W_{10}$ are defined by Eq. (6), $\Gamma_{01}$ and $\Gamma_{10}$ are defined by Eq. (11). Therefore, RII and the primary one would contribute to the final qubit population together. The population in state $|0\rangle$ is

$$
\begin{gathered}
p_{00}(t)=\frac{W_{10}\left(\varepsilon_{0}\right)+\Gamma_{10}\left(\varepsilon_{0}\right)}{\Gamma_{10}\left(\varepsilon_{0}\right)+2 W_{10}\left(\varepsilon_{0}\right)+\Gamma_{01}\left(\varepsilon_{0}\right)}+ \\
+\left[\tanh \left(\frac{\varepsilon_{0}}{2 T}\right)-\frac{W_{10}\left(\varepsilon_{0}\right)+\Gamma_{10}}{\Gamma_{10}\left(\varepsilon_{0}\right)+2 W_{10}\left(\varepsilon_{0}\right)+\Gamma_{01}\left(\varepsilon_{0}\right)}\right] \times \\
\times \exp \left\{-\left[\Gamma_{10}\left(\varepsilon_{0}\right)+2 W_{10}\left(\varepsilon_{0}\right)+\Gamma_{01}\left(\varepsilon_{0}\right)\right] t\right\} .
\end{gathered}
$$

Here we assume at the initial time the qubit is in equilibrium with its environment.

If the system dynamics time is long enough that the population is stationary, the population in state $|0\rangle$ can be described by

$$
p_{00}=\frac{W_{10}\left(\varepsilon_{0}\right)+\Gamma_{10}\left(\varepsilon_{0}\right)}{\Gamma_{10}\left(\varepsilon_{0}\right)+2 W_{10}\left(\varepsilon_{0}\right)+\Gamma_{01}\left(\varepsilon_{0}\right)} .
$$




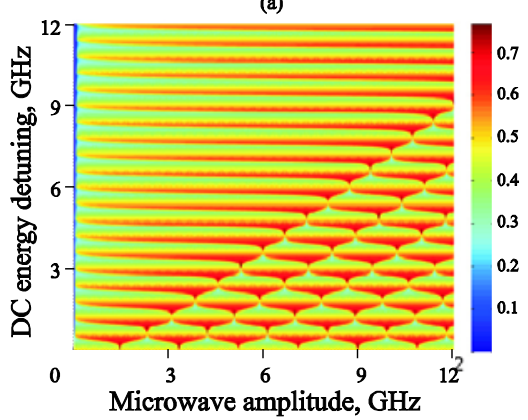

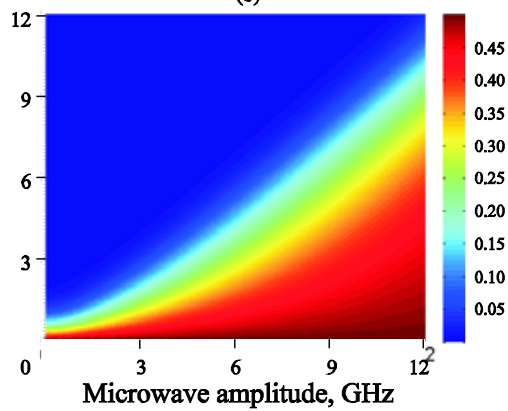

(c)

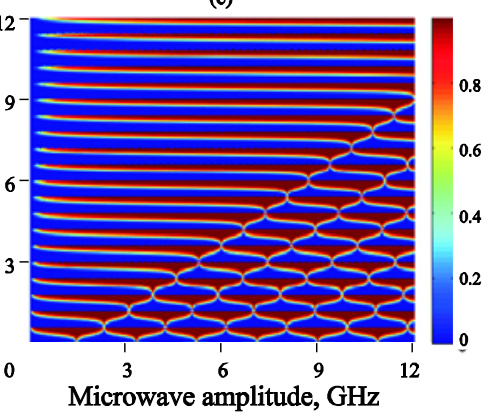

Fig. 3. (Color online) The stationary population of relaxation induced interference. The pattern is obtained from Eq. (15). (a) The characteristic frequency $\omega_{C} / 2 \pi=0.05 \mathrm{GHz}$ with the temperature $20 \mathrm{mK}$. Features of population inversion and periodical modulation are notable. (b) The characteristic frequency $\omega_{c} / 2 \pi=6 \mathrm{GHz}$ with the temperature $20 \mathrm{mK}$. (c) The characteristic frequency $\omega_{c} / 2 \pi=0.05 \mathrm{GHz}$ with the temperature $2 \cdot 10^{-5} \mathrm{mK}$. The driving frequency $\omega / 2 \pi=0.6 \mathrm{GHz}$.

RII, dependent on the characteristic frequency $\omega_{c}$, would greatly influence the final population, and hence the characteristic frequency $\omega_{c}$ would determine the final population distribution. $\Gamma_{10}\left(\Gamma_{01}\right)$ is on the order of $\omega_{c} \phi^{2} \alpha$, which is always smaller than $W_{10}\left(W_{01}\right)$ at the region $A>\varepsilon_{0}$. Therefore the stationary population is dominated by the symmetric transition rates $W_{10}$ and $W_{01}$ thus near- ly 0.5 . At the region $A<\varepsilon_{0}, W_{10}\left(W_{01}\right)$ can be neglected so that RII would dominate the population.

In the case $\omega \gg \omega_{c}$, for the increasing relaxation strength, i.e. from $\phi^{2} \alpha \omega_{c} \ll \Delta^{2} / \Gamma_{2}$ to $\phi^{2} \alpha \omega_{c} \sim \Delta^{2} / \Gamma_{2}$, at the region $A>\varepsilon_{0}$ population inversion can be more pronounced [Figs. 4(a) and (b)] while at the region $A<\varepsilon_{0}$ the population would be nearly 0.5 as indicated by Fig. 3(a). In
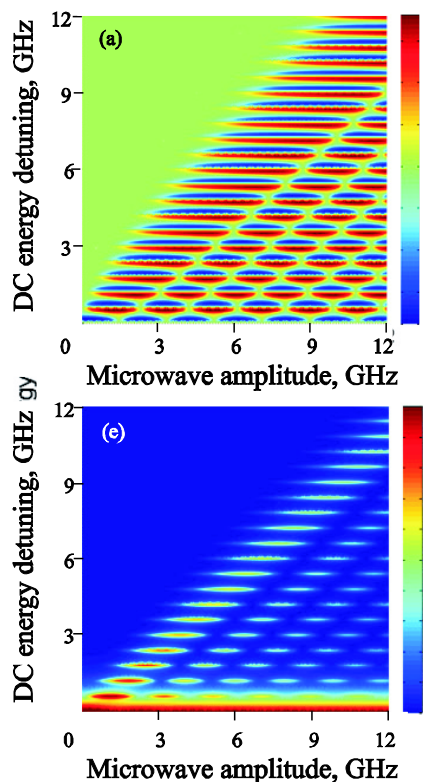
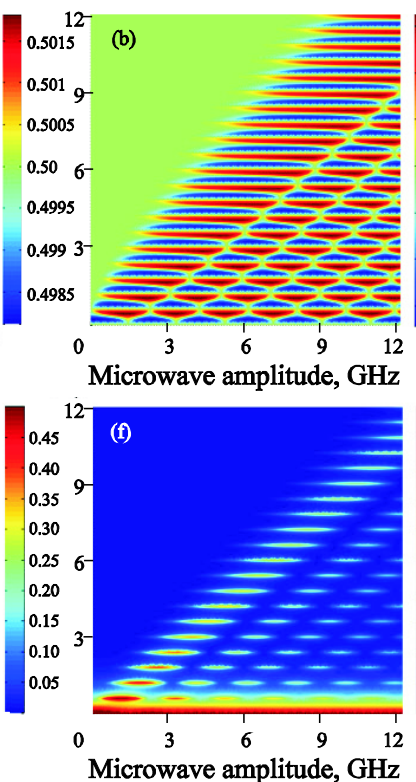
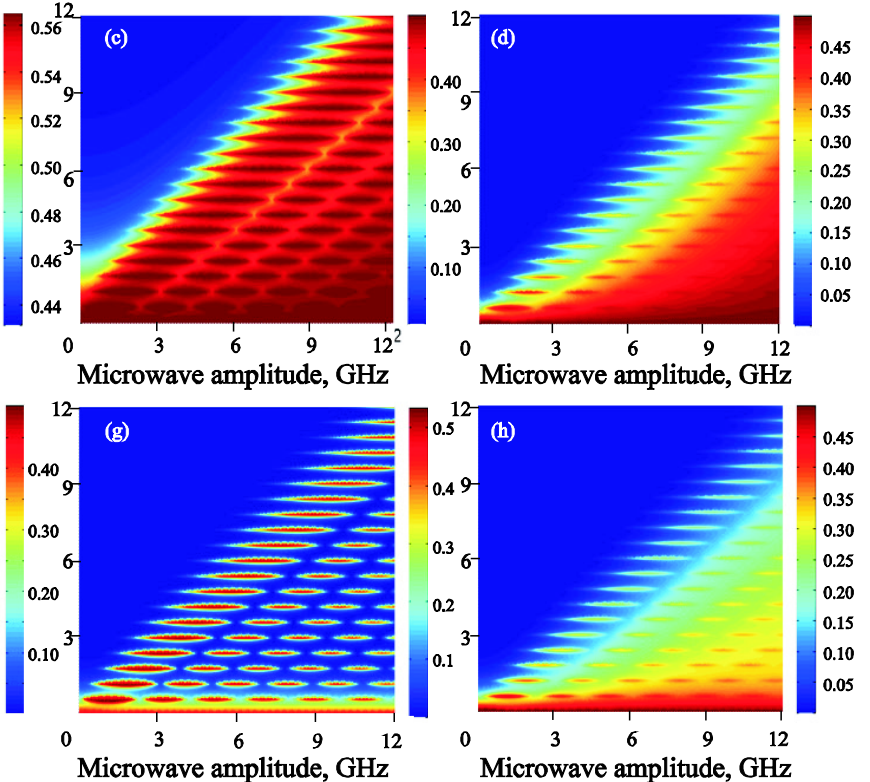

Fig. 4. (Color online) Calculated final qubit population versus energy detuning and microwave amplitude. (a) The stationary interference pattern in the weak relaxation situation. The parameters we used are the driving frequency $\omega / 2 \pi=0.6 \mathrm{GHz}$, the dephasing rate $\Gamma_{2} / 2 \pi=0.06 \mathrm{GHz}$, the couple tunneling $\Delta / 2 \pi=0.013 \mathrm{GHz}, \alpha \phi^{2}=0.0002$, the temperature is $20 \mathrm{mK}$, and the characteristic frequency $\omega_{C} / 2 \pi=0.05 \mathrm{GHz}$. The periodical patterns of RII can be seen, although not clear. (b) The stationary interference pattern in the strong relaxation situation with $\alpha \phi^{2}=0.02$ and $\omega_{C} / 2 \pi=0.05 \mathrm{GHz}$. Since the relaxation strength is stronger, the periodical interference patterns are more notable. (c) The stationary interference pattern in the weak relaxation situation with $\alpha \phi^{2}=0.000002$ and $\omega_{C} / 2 \pi=6 \mathrm{GHz}$. (d) The stationary interference pattern in the strong relaxation situation with $\alpha \phi^{2}=0.0002$ and $\omega_{C} / 2 \pi=6 \mathrm{GHz}$. (e) The unsaturated interference pattern in the weak relaxation situation. The system dynamics time $t=0.5 \mu$ s. The characteristic frequency $\omega_{C} / 2 \pi=0.05 \mathrm{GHz}, \alpha \phi^{2}=0.0002$. (f) The unsaturated interference pattern in the weak relaxation situation. The system dynamics time $t=0.5 \mu \mathrm{s}$. The characteristic frequency $\omega_{C} / 2 \pi=6 \mathrm{GHz}, \alpha \phi^{2}=0.000002$. (g) The unsaturated interference pattern in the strong relaxation situation. The system dynamics time $t=0.5 \mu \mathrm{s}$. The characteristic frequency $\omega_{c} / 2 \pi=0.05 \mathrm{GHz}, \alpha \phi^{2}=0.02$. (h) The unsaturated interference pattern in the strong relaxation situation. The dynamics time $t=0.5 \mu$ s. The characteristic frequency $\omega_{c} / 2 \pi=6 \mathrm{GHz}, \alpha \phi^{2}=0.0002$. The other parameters used in these figures are the same with those in Fig. 4(a). 
the case $\omega<\omega_{c}$, with the relaxation strength $\phi^{2} \alpha \omega_{c}$ increasing, at the region $A<\varepsilon_{0}$ the population would be nearly 0 as indicated by Fig. 3(b) while at the region $A>\varepsilon_{0}$ the visibility of RII improves, but with no population inversion, as shown in Figs. 4(c) and (d).

For the short system dynamics time, i.e. $t \sim \Gamma_{2} / \Delta^{2}$, the population in state $|0\rangle$ is described by Eq. (17). The relaxation strength determines the time the bath needs to excite the qubit, and becomes more important in determining the qubit population. For the weak relaxation strength, i.e. $\phi^{2} \alpha \omega_{c} \ll \Delta^{2} / \Gamma_{2}$, the bath has no enough time to excite the qubit. With the characteristic frequency increasing, there is little difference between interference patterns, as shown in Figs. 4(e) and (f). For the strong relaxation strength, i.e. $\phi^{2} \alpha \omega_{c} \sim \Delta^{2} / \Gamma_{2}$, RII emerges, and results in clearer difference between interference patterns for various characteristic frequencies [Figs. 4(g) and (h)].

Therefore, from the short time dynamics, we can obtain the information of the relaxation strength. Furthermore, from the long time dynamics, the effect of the characteristic frequency emerges.

\subsection{Phenomenological relaxation theory and discussions}

If we take an average approximation with $i A / \omega \sin \omega t_{1}-$ $-i A / \omega \sin \omega t_{2}$ in the second term of Eq. (A.5) in Appendix, the final expressions of transition rates in Eq. (7) will be changed to

$$
\Gamma_{10}=\frac{\phi^{2}}{4} S\left(-\varepsilon_{0}\right), \quad \Gamma_{01}=\frac{\phi^{2}}{4} S\left(\varepsilon_{0}\right) .
$$

Then assuming $\Gamma_{01}=$ const, we obtain $\Gamma_{10}=\Gamma_{01} \mathrm{e}^{-\varepsilon_{0} / T}$. This phenomenological theory is consistent with the existing experimental results [35]. Then we substitute Eq. (19) into Eqs. (17) and (18), obtaining the stationary and unsaturated population.

Now we discuss the valid region of the approximation. When the relaxation is weak, i.e. $\Gamma_{01} \ll \Delta^{2} / \Gamma_{2}$, the qubit population in state $|0\rangle$ in the short time dynamics $\left(t \approx \Gamma_{2} / \Delta^{2}\right)$ can be calculated as shown in Fig. 5(a). For the model of RII, the weak relaxation strength $\phi^{2} \alpha \omega_{c} \ll \Delta^{2} / \Gamma_{2}$ in the short time dynamics $\left(t \approx \Gamma_{2} / \Delta^{2}\right)$ would lead to the population in state $|0\rangle$ as shown in Figs. 4 (e) and (f). There is almost no difference between two theories in the weak relaxation and short time dynamics.

For the long driving time, the stationary situation will be reached. Shown in Fig. 5(b) is the population in state $|0\rangle$ obtained from the phenomenological theory. It is noticed that the stationary situation for the phenomenological theory does not agree with the long time dynamics of RII, as shown in Figs. 5(b), 4(a), and 4(c). But further from the detail comparison in Fig. 5(c) we can find that actually in an intermediary time scale the phenomenological theory is still a good approximation. However, if the time further increases, the difference between two models will be remarkable.

Then we consider the strong relaxation, i.e. $\Gamma_{01} \sim \Delta^{2} / \Gamma_{2}$. Although the relaxation is in the same order of magnitude with $\Delta^{2} / \Gamma_{2}$, it can still be much smaller than $\Gamma_{2}$. Shown in Figs. 5(d) and (e) are the results of the phenomenological theory in the short time dynamics
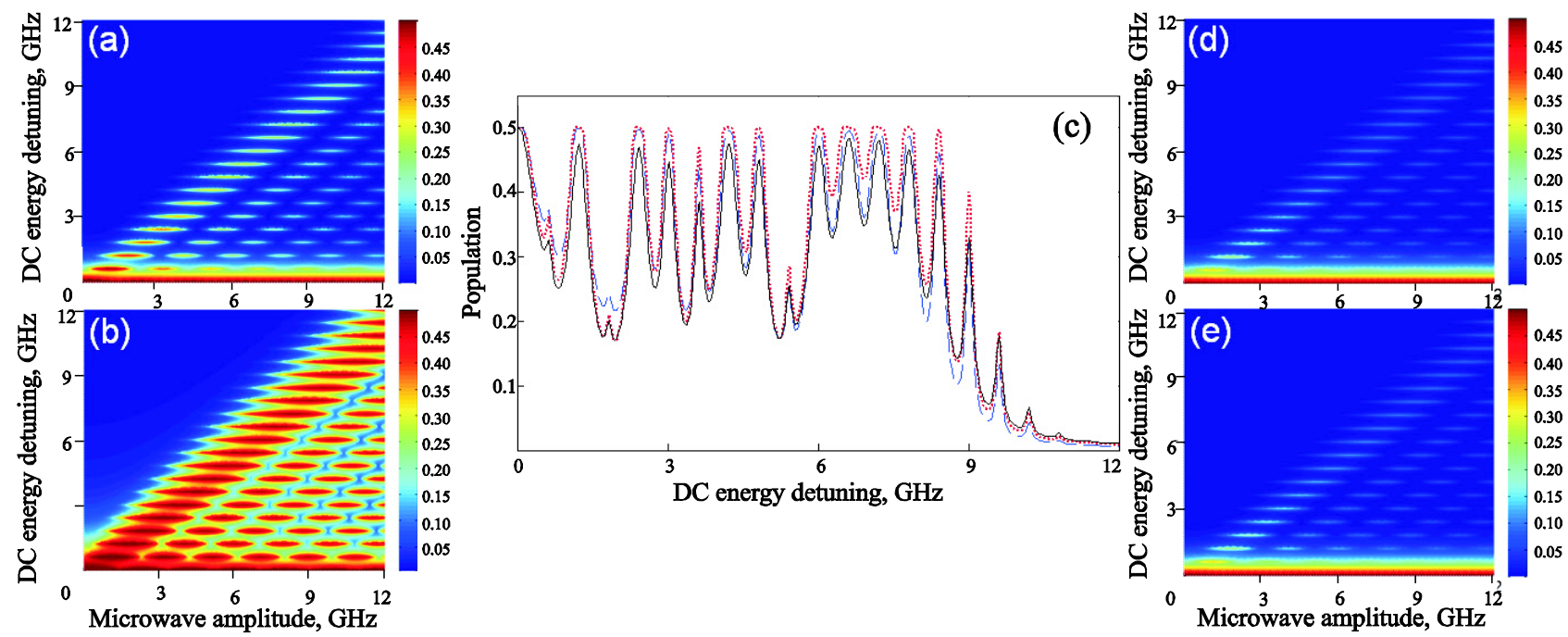

Fig. 5. (Color online) (a) The unsaturated interference pattern obtained by phenomenological relaxation theory. The system dynamics time is $0.5 \mu \mathrm{s}, \Gamma_{01} / 2 \pi=0.000008 \mathrm{GHz}$. (b) The stationary interference pattern obtained by the phenomenological relaxation theory. $\Gamma_{01} / 2 \pi=0.000008 \mathrm{GHz}$. (c) Comparison of the results of two theories. The blue dashed line expresses the population in $|0\rangle$ with the characteristic frequency $\omega_{C} / 2 \pi=6 \mathrm{GHz}$, the microwave amplitude is fixed at $8 \mathrm{GHz}, \phi^{2} \alpha=0.000002$ and the system dynamics time $t=13 \mu \mathrm{s}$, the red dotted line expresses the population with the characteristic frequency $\omega_{c} / 2 \pi=0.05 \mathrm{GHz}$, the microwave amplitude is fixed at $8 \mathrm{GHz}, \phi^{2} \alpha=0.0002$, and the system dynamics time $t=16 \mu \mathrm{s}$. The black line uses the stationary result with the phenomenological relaxation theory with $\Gamma_{01} / 2 \pi=0.000008 \mathrm{GHz}$, and the microwave amplitude is fixed at $8 \mathrm{GHz}$. (d) The unsaturated interference pattern obtained by phenomenological relaxation theory. The evolution time is $0.5 \mu \mathrm{s}, \Gamma_{01} / 2 \pi=0.001 \mathrm{GHz}$. (e) The stationary interference pattern obtained by phenomenological relaxation theory. $\Gamma_{01} / 2 \pi=0.001 \mathrm{GHz}$. Other parameters are identical with those of Fig. $4(\mathrm{a})$. 
$\left(t \approx \Gamma_{2} / \Delta^{2}\right)$ and in the stationary case, respectively. Comparing Fig. 5(d) with Figs. 4(g) and (h), we can find the disagreement of two models is large in the short time dynamics. In the stationary case, the disagreement is larger, as shown in Figs. 4(b), 4(d) and 5(e). This indicates that the phenomenological theory is only a good approximation of RII in the weak relaxation case.

On the other hand, the results of the phenomenological theory in Eq. (19) follow the Einstein relation with a larger downward relaxation rate. If we use Eqs. (6) and (19) in rate equations Eq. (16), larger downward relaxation rate would make more population exist at lower state and population inversion would not emerge. This is the result of well-known Einstein spontaneous and stimulated emission (ESSE) theory.

Actually, for the weak driving $A \ll \omega$, the transition rates Eq. (11) would be changed to the forms of Eq. (19). For the strong periodic driving, Eq. (19) would be replaced by Eq. (11). The relaxation described by Eq. (11) will form the asymmetric transitions and produce population inversion, breaking the Einstein relation, as well as ESSE theory. As shown in Figs. 3 and 4, at some detunings, the upward relaxation is larger than the downward one and the population at higher state is larger than 0.5 . Therefore, in the presence of the environment bath, ESSE theory is only justified in the weak driving. Since RII is not dependent on the crossover, these conclusions are applicable to a general TLS.

It should be mentioned Wilson et al. [47] demonstrated population inversion can occur in a TLS of a single Cooper-pair box in the dressed state picture. In their system, weak driving, not strong driving will produce population inversion. Furthermore, population inversion in their system is dependent on the tunnel coupling (crossover). Without the crossover structure, i.e. $\Delta=0$, population inversion will not emerge. These results are different from ours.

\section{Rabi-oscillation-induced interference}

In above sections, we investigate RII which is produced by the interaction of the qubit and electromagnetic bath. The bath can be considered as multimode electromagnetic fields. However, the characteristic frequency and coupling strength are dominated by the environment thus uncontrollable, which restrict the applications of EII. Therefore, in this section we will introduce a more controllable situation. For a clear physical picture, we apply the monochrome high-frequency weak field with only the longitudinal component, which would interact with the qubit as shown in Fig. 6. The resulted Rabi oscillations has been demonstrated in artificial mesoscopic systems, such as superconducting qubits, serve as a basic method to manipulate quantum states $[48,49]$.

In the following discussions, although the effect of the inevitable environment bath still needs to be considered, for a clear physical picture we will replace RII by the phe- nomenological relaxation theory discussed in Sec. 3, not treating it as multimode fields as discussed in former sections. Then the Hamiltonian (1) is changed to

$$
H=-\frac{\Delta}{2} \sigma_{X}-\frac{\varepsilon(t)}{2} \sigma_{z}-\frac{\tilde{\varepsilon}(t)}{2} \sigma_{z}-\frac{Q}{2} \sigma_{z}+H_{B},
$$

where $\tilde{\varepsilon}(t)=\tilde{A} \cos (\tilde{\omega} t)$ describes the weak field, $\tilde{A}$ and $\tilde{\omega}$ are the amplitude and frequency of the weak field. Now we make a transformation to an interaction picture with respect to $H_{B}-\tilde{\varepsilon}(t) \sigma_{z} / 2$. Therefore, the Hamiltonian is rewritten to

$$
\begin{gathered}
H_{2}=-\frac{\varepsilon(t)}{2} \sigma_{z}-\frac{Q(t)}{2} \sigma_{z}-\frac{\Delta}{2}\left[\sum J_{n}(\tilde{A} / \tilde{\omega}) \mathrm{e}^{-i n \tilde{\omega} t}\right] \sigma_{+}- \\
-\frac{\Delta}{2}\left[\sum J_{n}(\tilde{A} / \tilde{\omega}) \mathrm{e}^{i n \tilde{\omega} t}\right] \sigma_{-} .
\end{gathered}
$$

Due to the weak driving, i.e. $\tilde{A}<\tilde{\omega}$, we only consider the case $n= \pm 1$ in the sum of the above expression. Now the Hamiltonian is

$$
\begin{aligned}
H_{2}=-\frac{\varepsilon(t)}{2} \sigma_{z} & -\frac{Q(t)}{2} \sigma_{z}-\frac{\Delta J_{1}(\tilde{A} / \tilde{\omega})}{2}\left[\mathrm{e}^{-i \tilde{\omega} t}-\mathrm{e}^{i \tilde{\omega} t}\right] \sigma_{+}- \\
& -\frac{\Delta J_{1}(\tilde{A} / \tilde{\omega})}{2}\left[\mathrm{e}^{i \tilde{\omega} t}-\mathrm{e}^{-i \tilde{\omega} t}\right] \sigma_{-},
\end{aligned}
$$

where the last two terms would lead to familiar Rabi oscillations [42]. Then we repeat the process in Appendix. Considering the initial condition $\rho(0)=|1\rangle\langle 1|$, we have the population in state $|0\rangle$

$$
\begin{gathered}
\rho_{00}(t)=\frac{\Delta^{2} J_{1}^{2}(\tilde{A} / \tilde{\omega})}{4} \int_{0}^{t} d t_{1} \int_{0}^{t} d t_{2} \mathrm{e}^{i \int_{2}^{t_{2}} \varepsilon(\tau) d \tau} \times \\
\times \operatorname{Tr}_{B}\left\{\left\langle 0\left|\left\{\left[U_{-}^{\dagger}\left(t_{1}\right) U_{+}\left(t_{1}\right)\left(\mathrm{e}^{i \tilde{\omega} t_{1}}-\mathrm{e}^{-i \tilde{\omega} t_{1}}\right)\right] \sigma_{-}\right\}\right| 1\right\rangle\langle 1|\right. \\
\left.\otimes \rho_{B}\left\{\left[U_{+}^{\dagger}\left(t_{2}\right) U_{-}\left(t_{2}\right)\left(\mathrm{e}^{-i \tilde{\omega} t_{2}}-\mathrm{e}^{i \tilde{\omega} t_{2}}\right)\right] \sigma_{+}\right\}|0\rangle\right\} .
\end{gathered}
$$

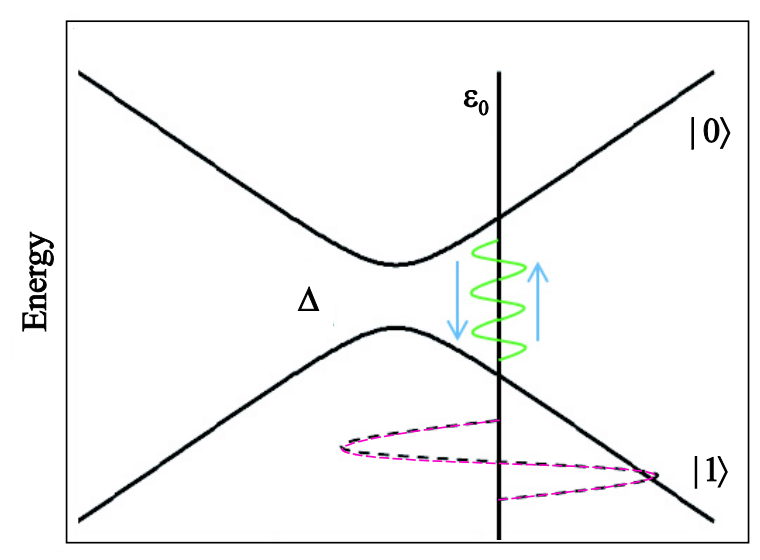

Energy detuning

Fig. 6. (Color online) Schematic energy diagram of a strongly driven two-level system interacting with a weak single-mode field. The green solid curve represents the weak field, forming effective coupling between states $|0\rangle$ and $|1\rangle$. 
We accept the rotating-wave approximation with the weak and strong fields, respectively, obtaining

$$
\begin{gathered}
\rho_{00}(t)=\frac{\Delta^{2} J_{1}^{2}(\tilde{A} / \tilde{\omega})}{4} \int_{0}^{t} d t_{1} \int_{0}^{t} d t_{2} \sum_{n} J_{n}^{2}\left(\frac{A}{\omega}\right) \times \\
\times \mathrm{e}^{i\left(\varepsilon_{0}+n \omega+\tilde{\omega}\right)\left(t_{1}-t_{2}\right)}\left\langle U_{+}^{\dagger}\left(t_{2}\right) U_{-}\left(t_{2}\right) U_{-}^{\dagger}\left(t_{1}\right) U_{+}\left(t_{1}\right)\right\rangle+ \\
+\frac{\Delta^{2} J_{1}^{2}(\tilde{A} / \tilde{\omega})}{4} \int_{0}^{t} d t_{1} \int_{0}^{t} d t_{2} \sum_{n} J_{n}^{2}\left(\frac{A}{\omega}\right) \times \\
\times \mathrm{e}^{i\left(\varepsilon_{0}+n \omega-\tilde{\omega}\right)\left(t_{1}-t_{2}\right)}\left\langle U_{+}^{\dagger}\left(t_{2}\right) U_{-}\left(t_{2}\right) U_{-}^{\dagger}\left(t_{1}\right) U_{+}\left(t_{1}\right)\right\rangle .
\end{gathered}
$$

If the noise spectral density is dominated by the white low-frequency noise, we have

$$
\begin{gathered}
\dot{\rho}_{00}(t)=\frac{\Delta^{2} J_{1}^{2}(\tilde{A} / \tilde{\omega})}{2} \sum_{n} \frac{\Gamma_{2} J_{n}^{2}(A / \omega)}{\left(\varepsilon_{0}+n \omega+\tilde{\omega}\right)^{2}+\Gamma_{2}^{2}}+ \\
+\frac{\Delta^{2} J_{1}^{2}(\tilde{A} / \tilde{\omega})}{2} \sum_{n} \frac{\Gamma_{2} J_{n}^{2}(A / \omega)}{\left(\varepsilon_{0}+n \omega-\tilde{\omega}\right)^{2}+\Gamma_{2}^{2}},
\end{gathered}
$$

where $\Gamma_{2}$ has the same definition with Eq. (6). Considering the small time interval, i.e. $\dot{\rho}_{00}(t) \approx W_{10}$, we obtain the transition rate from state $|1\rangle$ to $|0\rangle$

$$
\begin{gathered}
W_{10}=\frac{\Delta^{2} J_{1}^{2}(\tilde{A} / \tilde{\omega})}{2} \sum_{n} \frac{\Gamma_{2} J_{n}^{2}(A / \omega)}{\left(\varepsilon_{0}+n \omega+\tilde{\omega}\right)^{2}+\Gamma_{2}^{2}}+ \\
+\frac{\Delta^{2} J_{1}^{2}(\tilde{A} / \tilde{\omega})}{2} \sum_{n} \frac{\Gamma_{2} J_{n}^{2}(A / \omega)}{\left(\varepsilon_{0}+n \omega-\tilde{\omega}\right)^{2}+\Gamma_{2}^{2}},
\end{gathered}
$$

with $W_{01}\left(\varepsilon_{0}\right)=W_{01}\left(-\varepsilon_{0}\right)$. In order to ensure the validity of Eq. (26), the frequency of the weak field $\tilde{\omega}$ needs to be much larger than the driving frequency $\omega$ of the strong field.

In the absence of the strong field, i.e. $A=0$, the Hamiltonian in Eq. (20) is used to describe Rabi oscillations at the detunings $\varepsilon_{0}= \pm \tilde{\omega}$ with the Rabi frequency $\Delta J_{1}(\tilde{A} / \tilde{\omega})$. Rabi oscillations replaces the primary tunnel coupling $\Delta$ at the detunings $\varepsilon_{0}=0$ with two couplings at the detuning $A^{\prime}\left(\varepsilon_{0}=\tilde{\omega}\right)$ and $B^{\prime}\left(\varepsilon_{0}=-\tilde{\omega}\right), \Delta_{A^{\prime}}=\Delta_{B^{\prime}}=\Delta J_{1}(\tilde{A} / \tilde{\omega})$. Unlike the interwell relaxation, Rabi oscillations can construct symmetrical couplings between states $|1\rangle$ and $|0\rangle$, serving as a beam splitter working for both $|1\rangle$ and $|0\rangle$. Under the strong field the couplings at $A^{\prime}$ and $B^{\prime}$ can form effective crossovers to produce interferences, as shown in Figs. 7(a) and (b). We call this case Rabi-oscillationinduced interference. If $\tilde{\omega}>\Gamma_{2}$, the detunings $A^{\prime}$ and $B^{\prime}$ are well separated. It is important to notice that the position (a)
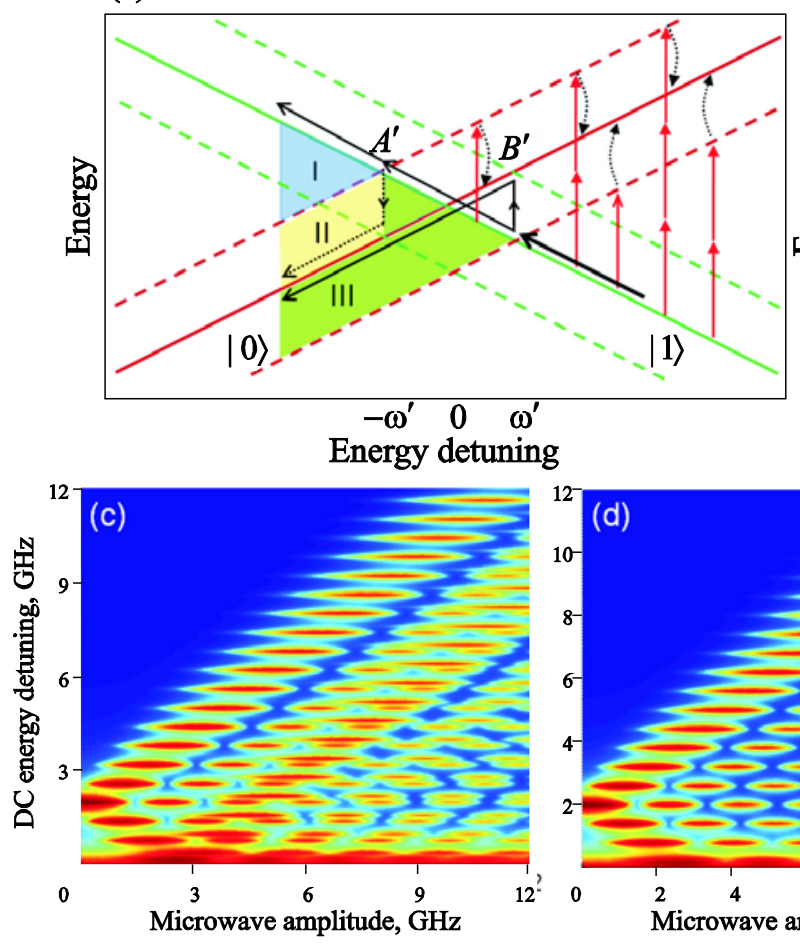

(b)

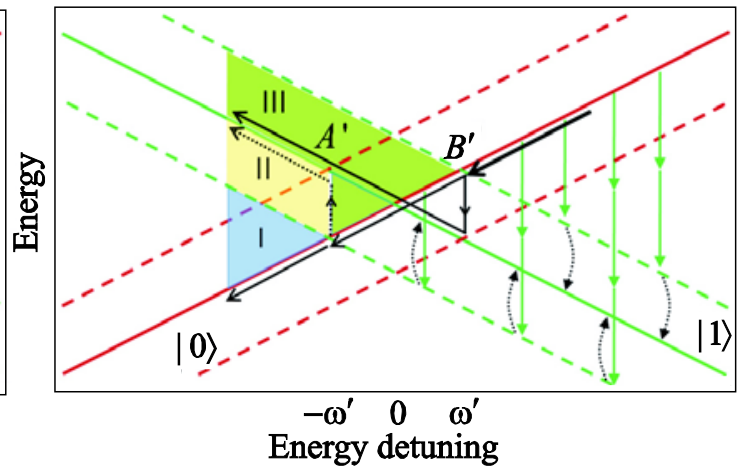

Fig. 7. (Color online) (a) and (b) Schematic energy diagram of Rabi-oscillation-induced interference: (a) describes the transition from state $|1\rangle$ to $|0\rangle$; (b) describes the transition from state $|0\rangle$ to $|1\rangle$. (c), (d), and (e) The interference pattern of population in state $|0\rangle$ obtained from Eqs. (26), (27), and (28), respectively. The parameters used here are $\omega / 2 \pi=2 \mathrm{GHz}, \tilde{A} / \omega=0.9, \quad \Gamma_{01} / 2 \pi=0.000008 \mathrm{GHz}$ and the temperature is $20 \mathrm{mK}$. Other parameters of the qubit are identical with Fig. 4(a). 
and strength of couplings can be controlled by the frequency and amplitude of the weak field. Moreover, the exponent forms in Eq. (24) are similar with that of Eq. (14). That means the weak field also produces a phase accumulation $i \tilde{\omega} t$, which would influence the interference phase and total phase accumulation, just as the multimode fields do.

First of all, we discuss the transition from state $|1\rangle$ to $|0\rangle$ [Fig. 7(a)]. When the initial state is $|1\rangle$, the microwave sweeps the state along $|1\rangle$ (black solid arrow). As reaching the detuning $B^{\prime}$, state $|1\rangle$ is split into the superposition of states $|1\rangle$ and $|0\rangle$, collecting different phases in two paths. When reaching the detuning $A^{\prime}$, state $|1\rangle$ is split into $|1\rangle$ and $|0\rangle$ again. Evolving independently, states $|1\rangle$ and $|0\rangle$ accumulate the same relative phase difference corresponding to couplings $A^{\prime}$ and $B^{\prime}$, marked with the region containing the blue region I, and the yellow region II. However, the effective phase difference contributing to the interference at $A^{\prime}$ is the blue region I since the weak field eliminates part of the phase (yellow region II). In a similar way, the effective phase difference contributing to the interference at $B^{\prime}$ is the region containing the blue region I, the yellow region II, and the green region III. The total phase $\theta_{A^{\prime}}$ gained over one period referring to $A^{\prime}$ is $2 \pi\left(\varepsilon_{0}+\tilde{\omega}\right) / \omega$, while the total phase $\theta_{B^{\prime}}$ gained over one period referring to $B^{\prime}$ is $2 \pi\left(-\varepsilon_{0}+\tilde{\omega}\right) / \omega$. The resonant transition from state $|1\rangle$ to $|0\rangle$ would occur at $\varepsilon_{0}=n \omega-\tilde{\omega}$ or $\varepsilon_{0}=n \omega+\tilde{\omega}$.

The transition from state $|0\rangle$ to $|1\rangle$ is similar [Fig. 7(b)]. The resonant transition from state $|0\rangle$ to $|1\rangle$ would occur at $\varepsilon_{0}=n \omega-\tilde{\omega}$ or $\varepsilon_{0}=n \omega+\tilde{\omega}$. This result is the same with that of transition from state $|0\rangle$ to $|1\rangle$, making that there is no population inversion in this case.

The energy conservation is satisfied for these transitions as shown in Fig. 7(a) and (b). For the transition from state $|1\rangle$ to $|0\rangle$, if the transition is through the coupling at $A^{\prime}$, besides $n$ photons with frequency $\omega$ absorbed, the total energy $n \omega=\varepsilon_{0}+\tilde{\omega}$ is larger than the energy spacing $\varepsilon_{0}$ so that the excess energy $\tilde{\omega}$ flows into the weak field. It is similar for other cases.

In order to describe the evolution of qubit population in the presence of two couplings, we employ rate equations, in which the qubit population obeys Eq. (16). But here, $W_{10}$ and $W_{01}$ are described by Eq. (26), with $\Gamma_{01} / 2 \pi=$ $=0.000008 \mathrm{GHz}, \quad \Gamma_{10}=\Gamma_{01} \mathrm{e}^{-\varepsilon_{0} / T}$, and the temperature $T=20 \mathrm{mK}$. Figure 7(c) is the calculated qubit population versus energy detuning and microwave amplitude. To understand this complicate pattern, firstly we consider the only effect of the coupling at $A^{\prime}$ with transition rates between states $|1\rangle$ and $|0\rangle$

$$
W_{10}=W_{01}=\frac{\Delta^{2} J_{1}^{2}(\tilde{A} / \tilde{\omega})}{2} \sum_{n} \frac{\Gamma_{2} J_{n}^{2}(A / \omega)}{\left(\varepsilon_{0}+n \omega-\tilde{\omega}\right)^{2}+\Gamma_{2}^{2}} .
$$

Substituting Eq. (27) into rate equations, we have the contour plot of the qubit population in state $|0\rangle$ as func- tions of energy detuning and microwave amplitude, shown in Fig. 7(d).

Then we only consider the effect of coupling at $B^{\prime}$ with transition rates between states $|1\rangle$ and $|0\rangle$

$$
W_{10}=W_{01}=\frac{\Delta^{2} J_{1}^{2}(\tilde{A} / \tilde{\omega})}{2} \sum_{n} \frac{\Gamma_{2} J_{n}^{2}(A / \omega)}{\left(\varepsilon_{0}+n \omega+\tilde{\omega}\right)^{2}+\Gamma_{2}^{2}} .
$$

With Eq. (28), we obtain the population in state $|0\rangle$ as shown in Fig. 7(e). Having addressed two couplings separately, we compare the results with Fig. 7(c) and find that they match very well. The accordance gives a clear physical picture that interferences based on couplings at $A^{\prime}$ and $B^{\prime}$ construct the final interference pattern.

Rabi oscillations changes the primary coupling at $\varepsilon_{0}=0$ to two couplings which are controllable in the position and coupling strength. Then two couplings result into ROII so as to form a complicate structure shown in Fig. 7(c). If we further increase the amplitude of the weak field, multiphoton Rabi oscillations would emerge and more couplings can be formed.

For EII, the longitudinal monochrome field forms the channels which depend on the tunnel coupling $\Delta$, such as Rabi oscillations in Eq. (26). On the contrary, the transverse multimode fields form the channels which are independent on $\Delta$, such as the interwell relaxation. Therefore, if we employ a transverse monochrome field, the qubitfield interaction will emerge in off-diagonal terms of the Hamiltonian Eq. (1) and Rabi oscillations can still be produced but independent on $\Delta$. As a result, even in the system without the double well structure, such as a phase qubit, ROII can still be produced.

\section{Superconducting qubits}

So far we have studied EII in an idealized TLS. We now consider the situation in the realistic system. An example of such case is a superconducting flux qubit, which have been studied considerably both theoretically and experimentally $[8,50]$.

The flux qubit is incorporated in a superconducting loop with three small-capacitance Josephson junctions. When the external magnetic flux bias through the loop $\Phi \approx 0.5 \Phi_{0}$, the classical potential energy has a double well structure parameterized by $\delta \Phi_{d c}=\Phi-0.5 \Phi_{0}$ and the kinetic part of Hamiltonian provides quantum tunnel coupling $\Delta$ between two wells. In the millikelvin temperature $\sim 20 \mathrm{mK}$, energy-level quantization emerges and the states localized in each well are separated by the energy intervals on the order of the plasma frequency $\omega_{p}$, which is usually much larger than $\Delta . \varepsilon_{0}=2 I_{p} \delta \Phi_{d c}$ is the dc energy detuning of states $|0\rangle$ and $|1\rangle$, with $I_{p}$ the maximum persistent current in the loop. The two lowest states in each well, $|0\rangle$ and $|1\rangle$, form an effective two-level system, which can be manipulated by application of microwave pulses. 
For a periodically driven flux qubit with the two-lowest states $|0\rangle$ and $|1\rangle$ in the right and left well, respectively, (Fig. 1) as we drive the qubit with the microwave $\Phi_{a c}=\Phi_{r f} \cos \omega t$, where $\Phi_{r f}$ is the microwave flux amplitude, the time-dependent magnetic flux $\delta \Phi(t)=\delta \Phi_{d c}+\Phi_{a c}$ controls the qubit and the time-dependent energy detuning of states $|0\rangle$ and $|1\rangle$ can be described as $\varepsilon(t)=\varepsilon_{0}+A \cos \omega t . A=2 I_{p} \Phi_{r f}$ is the microwave amplitude in the unit of magnetic flux. To produce ROII, we can apply another weak field as shown in Sec. 4, with extra external magnetic flux $\tilde{\Phi}=\tilde{\Phi}_{r f} \cos (\tilde{\omega} t)$ and $\tilde{A}=2 I_{p} \tilde{\Phi}_{r f}$. This is similar with circuit quantum electrodynamics (C-QED) where qubits are strongly coupled to quantum oscillators [51-53], especially the spectroscopy [54-56]. However C-QED is also hard to change the parameters and here the applied monochrome weak field can realize more flexible control.

In a recent experiment [57], two longitudinal strong monochrome fields were applied simultaneously on the flux qubit, just similar with the discussion in Sec. 4. Interesting phenomena different from our results were observed, since the amplitudes of two fields were modified together and there did not exist the stable coupling resulted by Rabi oscillations. Furthermore, the frequencies in two fields were comparable. Therefore, the phenomenon predicted here does not emerge in that experiment. In another experiment [17], two longitudinal weak monochrome fields were applied on the flux qubit. In this case, two fields interacted with the qubit and the energy transfer described above emerged. But the small amplitude made the interference we predicted invisible. Nevertheless, both experiments demonstrate the feasibility of our model.

ROII offers an alternative means to manipulate the qubit. It can be applied further in the time-dependent interference which can coherently manipulate the qubit state on the Bloch sphere with a single crossing [24,58] or continuous driving [20,57]. In addition, if the strong periodic driving is further replaced to the linear adiabatic passage, the interference is changed to Stark-chirped rapid adiabatic passages [59,60] which could serve as elementary logic gates for quantum computing. The adiabatic passages should be very slow compared to Rabi oscillations and fast compared to the coherence time. However, in our model, with a single crossing or continuous driving, the interference nature [18] allows faster manipulation than adiabatic passages, which is important for the short coherence time.

In addition, if we replace the multimode eletromagnetic field with the quantized single-mode field, such as microwave resonators [61], and optical cavity [62], the situation would be more interesting. Caused quantum noise would not only influence $\sigma_{X}$ to produce RII, but also have effects on $\sigma_{z}$ to cause unconventional Mach-Zehnder interference just as shown in Ref. 34. This case can be used to produce controllable cooling and population inversion.

\section{Conclusion}

We have proposed and comprehensively studied electromagnetically induced interference in quantum two-level systems based on the couplings formed by different electromagnetic systems.

First of all, we consider an electromagnetic bath which is familiar and unavoidable. We show the transverse multimode fields form the interwell relaxation, which can asymmetrically couple two qubit states at the detuning $\varepsilon_{0}= \pm \omega_{c}$. In the presence of the strong driving, the relaxation induced couplings can generate the interference, i.e. RII. Considering the rate equations, we show that the primary LZS interference resulted by $\Delta$ and RII interact together.

Our model provides a deeper understanding to the effect of the environment and shows the way to determine the relaxation strength and characteristic frequency of the environment bath. For the weak relaxation, we note that the phenomenological relaxation theory can be a good approximation. But for the strong relaxation, the phenomenological theory cannot be applicable. Moreover we show that RII can break the ESSE theory under the strong driving. As a result, population inversion can be produced in a strong driven TLS by the relaxation and the ESSE theory can only be justified under the weak driving.

Furthermore, we consider a longitudinal monochrome weak field, which interacts with the qubit through Rabi oscillations. We show Rabi oscillation changes the primary tunnel coupling into two symmetric beam splitters at $\varepsilon_{0}= \pm \tilde{\omega}$. Different from RII, ROII here can be controlled in the position and strength of the beam splitters by the amplitude and frequency of the weak field. ROII provides an alternative way to manipulate qubits in the strongly driven region, and characterize the qubit as well as the external fields. It provides the potential of further application in the time-dependent interference which can coherently manipulate the qubit state on the Bloch sphere with a single crossing or continuous driving. The models used here can be extended to other systems with double well such as quantum dots [22], or even without double well such as phase qubits. Relative experiments [58] have been already performed to test some predictions of this work.

\section{Appendix}

The system reduced density matrix can be expressed as

$$
\begin{gathered}
\rho(t)=\operatorname{Tr}_{B}\left[U_{1}(t, 0) \rho(0) \otimes \rho_{B} U_{1}^{\dagger}(t, 0)\right]= \\
=\operatorname{Tr}_{B}\left[\frac { 1 } { 4 } \int _ { 0 } ^ { t } d t _ { 1 } \int _ { 0 } ^ { t } d t _ { 2 } \left\{\left[\Delta U_{+}^{\dagger}\left(t_{1}\right) U_{-}\left(t_{1}\right)+\right.\right.\right. \\
\left.\left.+\phi U_{+}^{\dagger}\left(t_{1}\right) Q\left(t_{1}\right) U_{-}\left(t_{1}\right)\right] \mathrm{e}^{-i \int^{t_{1}} \varepsilon(\tau) d \tau}|1\rangle\langle 0|+\text { H.c. }\right\} \rho(0) \otimes
\end{gathered}
$$




$$
\begin{gathered}
\otimes \rho_{B}\left\{\left[\Delta U_{+}^{\dagger}\left(t_{2}\right) U_{-}\left(t_{2}\right)+\phi U_{+}^{\dagger}\left(t_{2}\right) Q\left(t_{2}\right) U_{-}\left(t_{2}\right)\right] \times\right. \\
\left.\left.\times \mathrm{e}^{-i \int^{0} \varepsilon(\tau) d \tau}|1\rangle\langle 0|+\text { H.c. }\right\}\right] .
\end{gathered}
$$

The strong dephasing makes the off-diagonal elements of $\rho(t)$ decay completely [37], and hence we focus on the diagonal part of the density matrix. Then we assume the initial condition $\rho(0)=|1\rangle\langle 1|$, and obtain $\rho_{00}(t)$

$$
\begin{gathered}
\rho_{00}(t)=\frac{1}{4} \int_{0}^{t} d t_{1} \int_{0}^{t} d t_{2} \operatorname{Tr}_{B}\left\{\langle 0 | \left\{\left[\Delta U_{-}^{\dagger}\left(t_{1}\right) U_{+}\left(t_{1}\right)+\right.\right.\right. \\
\left.\left.+\phi U_{-}^{\dagger}\left(t_{1}\right) Q\left(t_{1}\right) U_{+}\left(t_{1}\right)\right] \times \mathrm{e}^{i \int^{t_{1}} \varepsilon(\tau) d \tau}|0\rangle\langle 1|\right\}|1\rangle\langle 1| \otimes \\
\otimes \rho_{B}\left\{\left[\Delta U_{+}^{\dagger}\left(t_{2}\right) U_{-}\left(t_{2}\right)+\phi U_{+}^{\dagger}\left(t_{2}\right) Q\left(t_{2}\right) U_{-}\left(t_{2}\right)\right] \times\right. \\
=\frac{1}{4} \int_{0}^{t} d t_{1} \int_{0}^{t} d t_{2} \mathrm{e}^{t_{2}^{t_{2}} \varepsilon(\tau) d \tau} \quad\left\langle\mathrm{e}^{2} U_{+}^{\dagger}\left(t_{2}\right) U_{-}\left(t_{2}\right) U_{-}^{\dagger}\left(t_{1}\right) U_{+}\left(t_{1}\right)+\right. \\
+\phi^{2} U_{+}^{\dagger}\left(t_{2}\right) Q\left(t_{2}\right) U_{-}\left(t_{2}\right) U_{-}^{\dagger}\left(t_{1}\right) Q\left(t_{1}\right) U_{+}\left(t_{1}\right)+ \\
+\phi \Delta U_{+}^{\dagger}\left(t_{2}\right) U_{-}\left(t_{2}\right) U_{-}^{\dagger}\left(t_{1}\right) Q\left(t_{1}\right) U_{+}\left(t_{1}\right)+ \\
\left.+\Delta \phi U_{+}^{\dagger}\left(t_{2}\right) Q\left(t_{2}\right) U_{-}\left(t_{2}\right) U_{-}^{\dagger}\left(t_{1}\right) U_{+}\left(t_{1}\right)\right\rangle .
\end{gathered}
$$

We can calculate the first two average terms in Eq. (A.2) by expanding $U_{ \pm}^{\dagger}(t)$ to the second order in $Q(t)$, with

$$
\begin{gathered}
\left\langle U_{+}^{\dagger}\left(t_{2}\right) U_{-}\left(t_{2}\right) U_{-}^{\dagger}\left(t_{1}\right) U_{+}\left(t_{1}\right)\right\rangle \approx 1+ \\
+\frac{1}{2} \int_{t_{1}}^{t_{2}} d t^{\prime \prime}\left[\int_{0}^{t_{1}} d t^{\prime}\left\langle Q\left(t^{\prime \prime}\right) Q\left(t^{\prime}\right)\right\rangle-\int_{0}^{t_{2}} d t^{\prime}\left\langle Q\left(t^{\prime}\right) Q\left(t^{\prime \prime}\right)\right\rangle\right], \quad(\mathrm{A} .3) \\
\left\langle U_{+}^{\dagger}\left(t_{2}\right) Q\left(t_{2}\right) U_{-}\left(t_{2}\right) U_{-}^{\dagger}\left(t_{1}\right) Q\left(t_{1}\right) U_{+}\left(t_{1}\right)\right\rangle \approx\left\langle Q\left(t_{2}\right) Q\left(t_{1}\right)\right\rangle .
\end{gathered}
$$

Since the last two terms in Eq. (A.2) are neglected under the average approximation compared with the other two terms, we obtain

$$
\begin{aligned}
& \rho_{00}(t)=\frac{\Delta^{2}}{4} \int_{0}^{t} d t_{1} \int_{0}^{t} d t_{2}\left\langle U_{+}^{\dagger}\left(t_{2}\right) U_{-}\left(t_{2}\right) U_{-}^{\dagger}\left(t_{1}\right) U_{+}\left(t_{1}\right)\right\rangle \times \\
& \times \mathrm{e}^{i \int^{t_{2}} \varepsilon(\tau) d \tau}+\frac{\phi^{2}}{4} \int_{0}^{t} d t_{1} \int_{0}^{t} d t_{2} \mathrm{e}^{i \int_{2}^{t_{1}} \varepsilon(\tau) d \tau}\left\langle Q\left(t_{2}\right) Q\left(t_{1}\right)\right\rangle .
\end{aligned}
$$

The first term in Eq. (A.5) has been discussed in Ref. 35, where

$$
\left\langle U_{+}^{\dagger}\left(t_{2}\right) U_{-}\left(t_{2}\right) U_{-}^{\dagger}\left(t_{1}\right) U_{+}\left(t_{1}\right)\right\rangle=\exp \left\{\int d \omega^{\prime} S\left(\omega^{\prime}\right) \times\right.
$$

$$
\left.\times\left[\mathrm{e}^{i \omega^{\prime}\left(t_{1}-t_{2}\right)}-1-2 i \sin \frac{\omega^{\prime}\left(t_{1}-t_{2}\right)}{2} \cos \frac{\omega^{\prime}\left(t_{1}+t_{2}\right)}{2}\right] / \omega^{\prime 2}\right\} .
$$

If the noise is frequency independent (white) in low-frequency region $\omega^{\prime} / 2 \pi \lesssim 1 / t, S(\omega) \approx S(0)$, Eq. (A.6) can be integrated to yield

$$
\left\langle U_{+}^{\dagger}\left(t_{2}\right) U_{-}\left(t_{2}\right) U_{-}^{\dagger}\left(t_{1}\right) U_{+}\left(t_{1}\right)\right\rangle=\mathrm{e}^{-\Gamma_{2}\left|t_{2}-t_{1}\right|}
$$

where $\Gamma_{2}=\pi S(0)$. Then we focus on the second term in Eq. (A.5) which is related to the interwell relaxation. With the Bessel functions

$$
\mathrm{e}^{i\left(\frac{A}{\omega}\right) \sin \omega t}=\sum_{n} J_{n}\left(\frac{A}{\omega}\right) \mathrm{e}^{i n \omega t},
$$

where $J_{n}(A / \omega)$ are Bessel functions of the first kind, for the high frequency driving $\omega \gg \Delta J_{n}(A / \omega)$, where $n$ is the closest integer to $\varepsilon_{0} / \omega$, we apply the rotating-wave approximation to make

$$
\mathrm{e}^{i\left(\frac{A}{\omega}\right) \sin \omega t_{1}-i\left(\frac{A}{\omega}\right) \sin \omega t_{2}} \approx \sum_{n} J_{n}^{2}\left(\frac{A}{\omega}\right) \mathrm{e}^{i n \omega\left(t_{1}-t_{2}\right)},
$$

where we neglect the nonresonant terms with exponent oscillating quickly with high frequency comparing to the time scale of the dynamics $t \sim 1 /\left[\Delta J_{n}(A / \omega)\right]$. Hence we have

$$
\begin{aligned}
& \rho_{00}(t)= \frac{\Delta^{2}}{4} \int_{0}^{t} d t_{1} \int_{0}^{t} d t_{2} \sum_{n} J_{n}^{2}\left(\frac{A}{\omega}\right) \mathrm{e}^{i\left(\varepsilon_{0}+n \omega\right)\left(t_{1}-t_{2}\right)} \times \\
& \times\left\langle U_{+}^{\dagger}\left(t_{2}\right) U_{-}\left(t_{2}\right) U_{-}^{\dagger}\left(t_{1}\right) U_{+}\left(t_{1}\right)\right\rangle+ \\
&+\frac{\phi^{2}}{4} \int_{0}^{t} d t_{1} \int_{0}^{t} d t_{2} \sum_{n} J_{n}^{2}\left(\frac{A}{\omega}\right) \mathrm{e}^{i\left(\varepsilon_{0}+n \omega\right)\left(t_{1}-t_{2}\right)}\left\langle Q\left(t_{2}\right) Q\left(t_{1}\right)\right\rangle .
\end{aligned}
$$

With the inverse Fourier transformation

$$
\left\langle Q\left(t_{2}\right) Q\left(t_{1}\right)\right\rangle=\int d \omega^{\prime} \mathrm{e}^{-i \omega^{\prime}\left(t_{2}-t_{1}\right)} S\left(\omega^{\prime}\right),
$$

we find

$$
\begin{aligned}
& \rho_{00}(t)= \frac{\Delta^{2}}{4} \int_{0}^{t} d t_{1} \int_{0}^{t} d t_{2} \sum_{n} J_{n}^{2}\left(\frac{A}{\omega}\right) \mathrm{e}^{i\left(\varepsilon_{0}+n \omega\right)\left(t_{1}-t_{2}\right)} \times \\
& \times\left\langle U_{+}^{\dagger}\left(t_{2}\right) U_{-}\left(t_{2}\right) U_{-}^{\dagger}\left(t_{1}\right) U_{+}\left(t_{1}\right)\right\rangle+ \\
&+\frac{\phi^{2}}{4} \int_{0}^{t} d t_{1} \int_{0}^{t} d t_{2} \int d \omega^{\prime} \sum_{n} J_{n}^{2}\left(\frac{A}{\omega}\right) \mathrm{e}^{i\left(\varepsilon_{0}+n \omega+\omega^{\prime}\right)\left(t_{1}-t_{2}\right)} S\left(\omega^{\prime}\right) .
\end{aligned}
$$


For the time interval $t$, which is much larger than the dephasing time, we extend the integration limits $t$ to $\infty$ and obtain

$$
\begin{gathered}
\dot{\rho}_{00}(t)=\frac{\Delta^{2}}{4} \int_{-\infty}^{\infty} d \tau \sum_{n} J_{n}^{2}\left(\frac{A}{\omega}\right) \mathrm{e}^{i\left(\varepsilon_{0}+n \omega\right) \tau-\Gamma_{2}|\tau|}+ \\
+\frac{\phi^{2}}{4} \int_{-\infty}^{\infty} d \tau \sum_{n} J_{n}^{2}\left(\frac{A}{\omega}\right) \mathrm{e}^{i\left(\varepsilon_{0}+n \omega\right) \tau} \int d \omega^{\prime} e^{i \omega^{\prime} \tau} S\left(\omega^{\prime}\right)= \\
=\frac{\Delta^{2}}{2} \sum_{n} \frac{\Gamma_{2} J_{n}^{2}(A / \omega)}{\left(\varepsilon_{0}-n \omega\right)^{2}+\Gamma_{2}^{2}}+\frac{\phi^{2}}{4} \sum_{n} J_{n}^{2}\left(\frac{A}{\omega}\right) S\left(-\varepsilon_{0}-n \omega\right),
\end{gathered}
$$

where $\tau=t_{1}-t_{2}$.

\section{Acknowledgment}

Thanks to Xueda Wen for useful discussions. This work was supported in part by the State Key Program for Basic Researches of China (2011CB922104, 2011CBA00205), the NSFC (91021003, 11274156), the Natural Science Foundation of Jiangsu Province (BK2010012), and PAPD.

1. M.S. Child, Molecular Collision Theory, Academic Press, London (1974).

2. E.E. Nikitin and S.Ya. Umanskii, Theory of Slow Atomic Collisions, Springer, Berlin (1984).

3. N.B. Delone and V.P. Krainov, Atoms in Strong Light Fields, Springer-Verlag, Berlin, Springer Series in Chemical Physics, vol. 28 (1985).

4. R.J.C. Spreeuw, N.J. van Druten, M.W. Beijersbergen, E.R. Eliel, and J.P. Woerdman, Phys. Rev. Lett. 65, 2642 (1990); D. Bouwmeester, N.H. Dekker, F.E.V. Dorsselaer, C.A. Schrama, P.M. Visser, and J.P. Woerdman, Phys. Rev. A 51, 646 (1995).

5. M. Mark, T. Kraemer, P. Waldburger, J. Herbig, C. Chin, H.-C. Nägerl, and R. Grimm, Phys. Rev. Lett. 99, 113201 (2007); M. Mark, F. Ferlaino, S. Knoop, J.G. Danzl, T. Kraemer, C. Chin, H.-C. Nägerl, and R. Grimm, Phys. Rev. A 76, 042514 (2007).

6. D. Witthaut, E.M. Graefe, and H.J. Korsch, Phys. Rev. A 73, 063609 (2006); Q. Zhang, P. Hänggi, and J.B. Gong, Phys. Rev. A 77, 053607 (2008).

7. G. Wendin and V.S. Shumeiko, Low Temp. Phys. 33, 724 (2007).

8. Y. Makhlin, G. Schцn, and A. Shnirman, Rev. Mod. Phys. 73, 357 (2001).

9. M.A. Nielsen and I.L. Chuang, Quantum Computation and Quantum Information, Cambridge University Press, Cambridge (2000).

10. L.D. Landau, Phys. Z. Sowjetunion 2, 46 (1932); G. Zener, Proc. R. Soc. London. Ser. A 137, 696 (1932); E.C.G. Stückelberg, Helv. Phys. Acta 5, 369 (1932).

11. J. Ankerhold and H. Grabert, Phys. Rev. Lett. 91, 016803 (2003).
12. G. Ithier, E. Collin, P. Joyez, D. Vion, D. Esteve, J. Ankerhold, and H. Grabert, Phys. Rev. Lett. 94, 057004 (2005).

13. K. Saito, M. Wubs, S. Kohler, P. Hanggi, and Y. Kayanuma, Europhys. Lett. 76, 22 (2006).

14. K. Saito and Y. Kayanuma, Phys. Rev. B 70, 201304(R) (2004).

15. A.V. Shytov, D.A. Ivanov, and M.V. Feigel'man, Eur. Phys. J. B 36, 263 (2003).

16. S.N. Shevchenko, S. Ashhab, and F. Nori, Phys. Rep. 492, 1 (2010).

17. S. Saito, T. Meno, M. Ueda, H. Tanaka, K. Semba, and H. Takayanagi, Phys. Rev. Lett. 96, 107001(2006).

18. W.D. Oliver, Y. Yu, J.C. Lee, K.K. Berggren, L.S. Levitov, and T.P. Orlando, Science 310, 1653 (2005).

19. M. Sillanpää, T. Lehtinen, A. Paila, Y. Makhlin, and P. Hakonen, Phys. Rev. Lett. 96, 187002(2006).

20. D.M. Berns, M.S. Rudner, S.O. Valenzuela, K.K. Berggren, W.D. Oliver, L.S. Levitov, and T.P. Orlando, Nature (London) 455, 51(2008).

21. S.N. Shevchenko, A.N. Omelyanchouk, and E. Ilichev, Fiz. Nizk. Temp. 38, 360 (2012)] [Low Temp. Phys. 38, 283 (2012)].

22. J.R. Petta, H. Lu, and A.C. Gossard, Science 327, 669 (2010).

23. M.D. LaHaye, J. Suh, P.M. Echternach, K.C. Schwab, and M.L. Roukes, Nature 459, 960 (2009).

24. G.Z. Sun, X.D. Wen, B. Mao, Z.Y. Zhou, Y. Yu, P.H. Wu, S.Y. Han, Nature Commun. 1:51, doi: 10.1038/ncomms1050 (2010).

25. G.Z. Sun, X.D. Wen, Y.W. Wang, S.H. Cong, J. Chen, L. Kang, W.W. Xu, Y. Yu, S.Y. Han, and P.H. Wu, Appl. Phys. Lett. 94, 102502 (2009).

26. Y.W. Wang, S.H. Cong, X.D. Wen, C. Pan, G.Z. Sun, J. Chen, L. Kang, W.W. Xu, Y. Yu, and P.H. Wu, Phys. Rev. B 81, 144505 (2010).

27. C.M. Wilson, T. Duty, F. Persson, M. Sandberg, G. Johansson, and P. Delsing, Phys. Rev. Lett. 98, 257003 (2007).

28. A. Izmalkov, S.H.W. van der Ploeg, S.N. Shevchenko, M. Grajcar, E. Il'ichev, U. Hübner, A.N. Omelyanchouk, and H.-G. Meyer, Phys. Rev. Lett. 101, 017003 (2008).

29. A.J. Leggett, S. Chakravarty, A.T. Dorsey, M.P.A. Fisher, A. Garg, and W. Zwerger, Rev. Mod. Phys. 59, 1 (1987).

30. L. Tian, S. Lloyd, and T.P. Orlando, Phys. Rev. B 65, 144516 (2002).

31. C.H. Van der Wal, F.K. Wilhelm, C.J.P.M. Harmans, and J.E. Mooij, Eur. Phys. J. B 31, 111 (2003).

32. R.W. Simmonds, K.M. Lang, D.A. Hite, S. Nam, D.P. Pappas, and J.M. Martinis, Phys. Rev. Lett. 93, 077003 (2004).

33. K.B. Cooper, M. Steffen, R. McDermott, R.W. Simmonds, S. Oh, D.A. Hite, D.P. Pappas, and J.M. Martinis, Phys. Rev. Lett. 93, 180401 (2004).

34. J.M. Martinis, K.B. Cooper, R. McDermott, M. Steffen, M. Ansmann, K.D. Osborn, K. Cicak, S. Oh, D.P. Pappas, R.W. Simmonds, and C.C. Yu, Phys. Rev. Lett. 95, 210503 (2005).

35. D.M. Berns, W.D. Oliver, S.O. Valenzuela, A.V. Shytov, K.K. Berggren, L.S. Levitov, and T.P. Orlando, Phys. Rev. Lett. 97, 150520(2006).

36. L.J. Du, M.J. Wang, and Y. Yu, Phys. Rev. B 82, 045128 (2010).

37. L.J. Du, and Y. Yu, Phys. Rev. B 82, 144524 (2010). 
38. M.H.S. Amin and D.V. Averin, Phys. Rev. Lett. 100, 197001 (2008).

39. M.H.S. Amin and F. Brito, Phys. Rev. B 80, 214302(2009).

40. I. Goychuk and P. Hänggi, Adv. Phys. 54, 525 (2005).

41. M. Grifoni and P. Hänggi, Phys. Rep. 304, 229 (1998).

42. S. Ashhab, J.R. Johansson, A.M. Zagoskin, and F. Nori, Phys. Rev. A 75, 063414 (2007).

43. Y. Gefen, E. Ben-Jacob, and A.O. Caldeira, Phys. Rev. B 36, 2770 (1987).

44. S.N. Shevchenko, A.S. Kiyko, A.N. Omelyanchouk, and W. Krech, Low Temp. Phys. 31, 569 (2005).

45. K. Saito, M. Wubs, S. Kohler, Y. Kayanuma, and P. Hanggi, Phys. Rev. B 75, 214308 (2007).

46. J.M. Martinis, S. Nam, J. Aumentado, K.M. Lang, and C. Urbina, Phys. Rev. B 67, 094510 (2003).

47. C.M. Wilson, G. Johansson, T. Duty, F. Persson, M. Sandberg, and P. Delsing, Phys. Rev. B 81, 024520 (2010).

48. Y. Nakamura, Y.A. Pashkin, and J.S. Tsai, Phys. Rev. Lett. 87, 246601 (2001);

49. Y. Nakamura, Y.A. Pashkin, and J.S. Tsai, Nature 398, 786 (1999); D. Vion, A. Aassime, A. Cottet, P. Joyez, H. Pothier, C. Urbina, D. Esteve, and M.H. Devoret, Science 296, 886 (2002); Y. Yu, S.Y. Han, X. Chu, S.-I. Chu, and Z. Wang, Science 296, 889 (2002); J.M. Martinis, S. Nam, J. Aumentado, and C. Urbina, Phys. Rev. Lett. 89, 117901 (2002); I. Chiorescu, Y. Nakamura, C.J.P.M. Harmans, and J.E. Mooij, Science 299, 1869 (2003).
50. J.E. Mooij, T.P. Orlando, L. Levitov, L. Tian, C.H. van der Wal, and S. Lloyd, Science 285, 1036 (1999).

51. I. Chiorescu, P. Bertet, K. Semba, Y. Nakamura, C.J.P.M. Harmans, and J.E. Mooij, Nature (London) 431, 159 (2004).

52. A. Wallraff, D.I. Schuster, A. Blais, L. Frunzio, R.-S. Huang, J. Majer, S. Kumar, S.M. Girvin, and R.J. Schoelkopf, Nature (London) 431, 162 (2004).

53. R.J. Schoelkopf and S.M. Girvin, Nature 451, 664 (2008).

54. A. Fedorov, A.K. Feofanov, P. Macha, P. Forn-Dĺaz, C.J.P.M. Harmans, and J.E. Mooij, Phys. Rev. Lett. 105, 060503 (2010).

55. J. Johansson, S. Saito, T. Meno, H. Nakano, M. Ueda, K. Semba, and H. Takayanagi, Phys. Rev. Lett. 96, 127006 (2006).

56. F. Deppe, M. Mariantoni, E.P. Menzel, A. Marx, S. Saito, K. Kakuyanagi, H. Tanaka, T. Meno, K. Semba, H. Takayanagi, E. Solano, and R. Gross, Nature Physics 4, 686 (2008).

57. J. Bylander, M.S. Rudner, A.V. Shytov, S.O. Valenzuela, D.M. Berns, K.K. Berggren, L.S. Levitov, and W.D. Oliver, Phys. Rev. B 80, 220506 (2009).

58. G.Z. Sun, X.D. Wen, B. Mao, Y. Yu, J. Chen, W.W. Xu, L. Kang, P.H. Wu, and S.Y. Han, Phys. Rev. B 83, 180507 (2011).

59. M.M.T. Loy, Phys. Rev. Lett. 32, 814 (1974).

60. L.F. Wei, J.R. Johansson, L.X. Cen, S. Ashhab, and F. Nori, Phys. Rev. Lett. 100, 113601 (2008).

61. R.J. Schoelkopf and S.M. Girvin, Nature 451, 664 (2008).

62. F. Marquardt, J.P. Chen, A.A. Clerk, and S.M. Girvin, Phys. Rev. Lett. 99, 093902 (2007). 